\title{
Testing the sensory hypothesis of the early left anterior negativity with auditory stimuli
}

\author{
Evan D. Bradley and Arild Hestvik \\ Department of Linguistics and Cognitive Science, University of Delaware
}

\section{INTRODUCTION}

The Early Left Anterior Negativity (ELAN) is an event-related potential (ERP) component associated with syntactic processing and characterized by a negative-going wave in left anterior electrodes as early as $120 \mathrm{~ms}$ after an unexpected of word category (Friederici et al., 1993, inter alia). Friederici (2002) proposed a model of processing in which syntactic structure is built based on grammatical categories, before a semantic phase in which semantic content and argument structure relations are computed. Under this model, ELAN is assumed to be generated by left-anterior brain regions in the early syntax-only phase when an unexpected word category interferes with phrase structure building. Not yet fully explained is how a syntactic violation can be diagnosed so early by frontal brain regions, when the ELAN occurs in the same time range as early sensory processing (Hickok \& Poeppel, 2007, inter alia). Recent work (Lau et al. 2006; Dikker et al., 2009) has led to a hypothesis that the ELAN has a greater basis in sensory processing than previously assumed. The present study aims to test this sensory hypothesis in the auditory domain.

Lau et al. (2006) explained the early occurrence of the ELAN as a function of expectancy, rather than ungrammaticality per se. As the parser builds syntactic structure based on each word encountered in an utterance, upcoming grammatical categories are anticipated. Dikker et al. (2009) proposed that because the ELAN occurs in the same time period as early sensory processing, it is in fact a response to a violation of grammatically-generated expectations about physical properties generated by sensory areas rather than higher-level structure-building areas. They note that previous ELAN results were demonstrated only with targets including overt, closed-class functional morphology (e.g., (1) from Friederici et al. (1993), containing the German participle $g e-$ ).

(1) * Das Baby wurde im gefüttert the baby was in the fed

Dikker et al. (2009) found that the visual M100, a MEG component generated by occipital visual cortex in the ELAN time range, was more negative after an unexpected item, but only when the unexpected item contained a closed-class morpheme(2 vs. 3); no response to unexpected items was found in anterior (non-sensory) regions. Dikker and colleagues concluded that the visual analogue of the ELAN in MEG is a deflection of the M100 component, which is indeed generated by sensory areas, specifically the visual cortex for visually presented sentences, and that it reflects the detection of visual properties of closed-class function morphemes which are unexpected in the context of a strong top-down anticipation of a word's grammatical category.

Thanks are due to Ann Bunger and Laura Evans for help constructing the stimuli; to Catherine Bradley for assistance aquiring the data; to Suzanne Dikker, Lisa Rosenfelt, and others at the CUNY 2009 meeting for fruitful discussion. 
(2) * The discovery was in the reported

(3) * The discovery was report

The present study aims to examine the sensory ELAN hypothesis in the auditory domain by attempting to verify whether previous visual MEG results generalize to EEG components elicited by auditory sentences. Specifically, does the presence of closed-class morphology modulate the ELAN response to syntactic category violations during real-time auditory sentence processing?

If auditory cortex differentiates between categories based on form typicality enhanced by morphology, then ELAN will occur for category violations which are signaled by a functional morpheme (4) and not for those which lack it (5), while unexpected targets without such morphology cannot be diagnosed as quickly, and will be indexed by a later component; if ELAN indexes general failure of syntactic structure building, a similar response to each target is expected.

(4) * The dog that the cat kissed the turtle on the nose ran far away

(5) * The dog that the cat kissed turtles on the nose ran far away

\section{EXPERIMENT 1}

Experiment 1 examined ERP responses to filled-gap sentences (6, UngramObj) which were previously shown to elicit ELAN vs. control (7, AdjRel) (Hestvik et al., 2007). The frequency of each sentence type was $16.7 \%$ including fillers, and sentences were synthesized using ModelTalker text-to-speech system.

(6) * The dog that the cat kissed the turtle on the nose ran far away

(7) The day that the cat kissed the turtle on the nose, they ran far away

18 adults (10 female, age 19-28) responded to two-alternative forced-choice comprehension questions following auditory sentence presentation using E-Prime during 128-channel, 250Hz EEG acquisition at $250 \mathrm{~Hz}$. Each participant responded to 64 trials each in test and control conditions, and 384 total trials were collected per subject, including fillers. EEG was segmented into $1000 \mathrm{~ms}$ epochs with a $200 \mathrm{~ms}$ baseline period synchronized to the onset of the first word following the relative clause verb. Bad channels replaced using spherical spline interpolation, baselines corrected, and trials containing eye blinks or artifacts discarded; two subjects were eliminated due to excessive artifacts ( $>50 \%$ of trials).

UngramObj targets elicited a more negative-going wave in anterior electrodes compared to AdjRel targets, which was strongest at frontal electrodes 1 and 25 (figure 1). The waveforms of the two conditions diverge around $240 \mathrm{~ms}$ after the target, followed by a sustained negativity lasting the duration of the epoch. Analysis of variance reveals a significant main effect of condition on the amplitude of electrode 25 between 240 and $440 \mathrm{~ms}$ post-target $(F(1,348)=4.636, p<.05)$. The latency of this component is longer than that typical described for ELAN; this component is more consistent with the Left Anterior Negativity (LAN), associated with syntactic dependency resolution (Kluender \& Kutas, 1993).

UngramObj targets also elicited a more positive-going wave in fronto-central electrodes near the vertex compared to AdjRel targets, which was strongest at left-frontal electrode 7 (figure 2). The waveforms of the two conditions diverge around $500 \mathrm{~ms}$ after the target. Analysis of variance reveals a significant main effect of condition on the amplitude of electrode 7 between 500 and $700 \mathrm{~ms}$ post-target $(F(1,1886)=$ 88.466, $p<.001)$. This component is consistent with the P600, associated with syntactic reanalysis and repair.

\section{EXPERIMENT 2}

Experiment 2 used a procedure and analysis identical to that of Experiment 1. Stimuli for Experiment 2 consisted of Experiment 1 sentences with a plural NP in the gap position, instead of a the NP (8, 9). 17 adults (10 female, age 19-38) participated in Experiment 2; three were eliminated due to artifacts. 

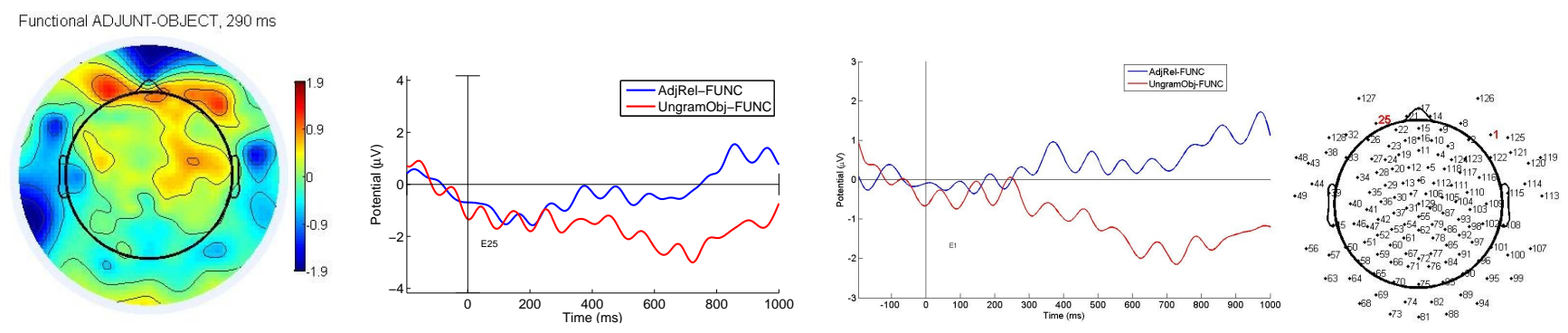

Figure 1. AdjRel-UngramObj scalp plot at $290 \mathrm{~ms}$ post-target and waveforms at electrodes 25 (left) and 1 (right; see inset for locations) in Experiment 1.
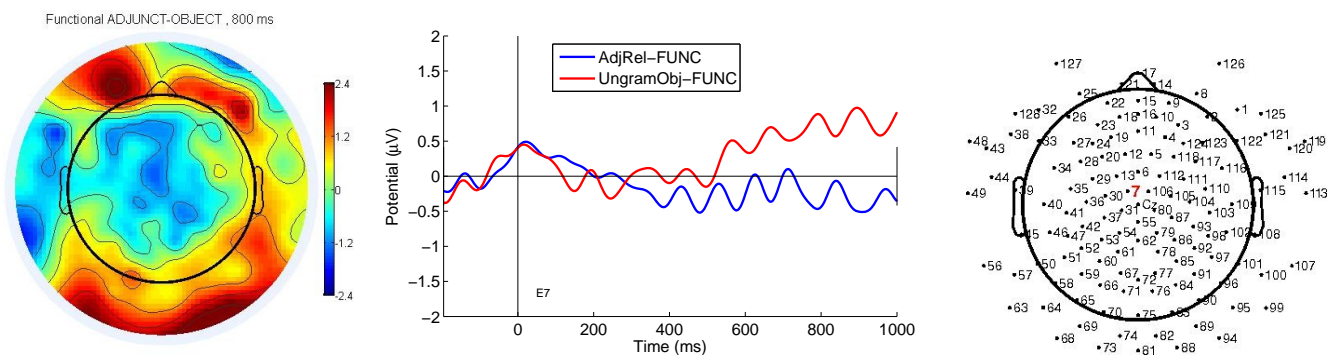

Figure 2. AdjRel-UngramObj scalp plot at $800 \mathrm{~ms}$ and waveform at electrode 7 (see inset for location) in Experiment 1 .

(8) * The dog that the cat kissed turtles on the nose ran far away

(9) The day that the cat kissed turtles on the nose, they ran far away

UngramObj targets elicited a broad negativity in centro-parietal electrodes (figure 3). In peak electrode 62, this negativity peaks around 400ms post-stimulus (figure ??). Analysis of variance reveals a significant main effect of condition on the amplitude of four midline electrodes between 300 and $500 \mathrm{~ms}$ post-target $(F(1,1424)=158.213, p<.001)$. consistent with N400 associated with semantic integration suggests processing of content, as well as category

\section{DISCUSSION}

The failure to replicate previous results which found ELAN for sentences like those in Experiment 1 (Hestvik et al., 2007) could be due in part to the use of synthetic stimuli, which either reduced the amplitude or increased the latency (or both) of the ELAN, possibly because of the extra attention required to comprehend the stimuli, which modulates the degree of expectation created by the sentences. The components found in Experiment 1 are consistent with a parser which generates a structural expectation
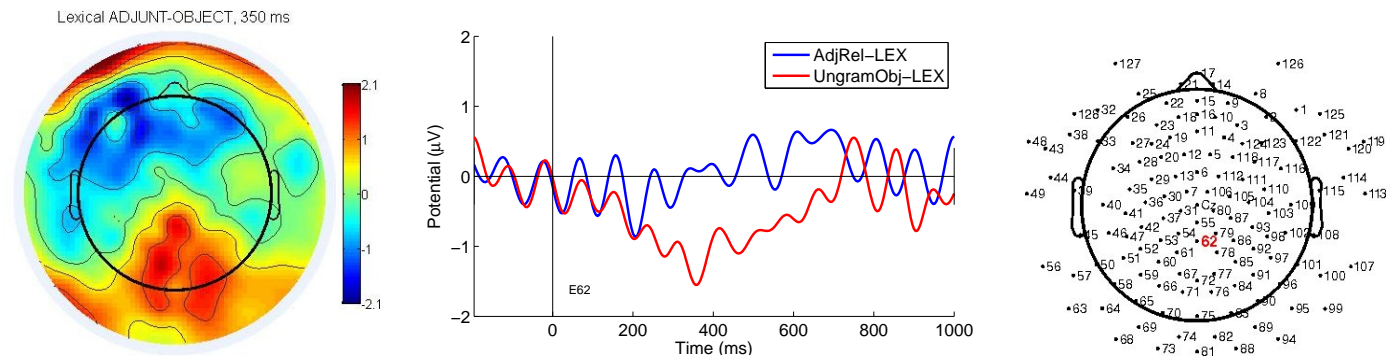

Figure 3. AdjRel-UngramObj scalp plot at $800 \mathrm{~ms}$ and waveform at electrode 62 (see inset for location) in Experiment 2. 
and diagnoses an unexpected grammatical category at an early stage; the filler-gap dependency cannot be computed with this unexpected item (LAN), leading to attempted resolution of the ambiguity of having too many possible fillers at a late stage (P600).

Contrary to expectation, unexpected targets in Experiment 2 did not elicit later syntactic components (LAN or P600). It was predicted that because these unexpected NPs violate the rules of relative clause phrase structure, they should be indexed by syntactic components such as LAN and P600 even if the NPs lack the morphology to allow the early diagnosis indexed by the ELAN. Instead, unexpected plurals elicited N400, suggesting that the content, as well as the structure, of these sentences is processed, and that the N400 reflects difficulty incorporating the 'extra' NP into argument structure. In other words, the ungrammatical NP is not perceived as a structural violation during the earliest processing stage associated with the ELAN (and these targets are not in fact violations of strictly local phrase structure).

We speculate that such morphology facilitates faster recognition of syntactic errors, so these sentences are abandoned as soon as offending morphology is encountered, with no attempt at argument structure integration. Without overt morphology, category is less extractable from form, so once the listener hears enough of the word to interpret category, the whole word can be understood, and semantic integration is attempted anyway (and fails).

\section{SUMMARY}

During auditory sentence processing, filled-gap NPs introduced by overt closed-class functional morphology elicit syntactic components, while filled-gap NPs without such morphology elicit a later semantic component. These results support components of the sensory ELAN hypothesis in the auditory domain to the extent that an earlier response is elicited when the target is introduced by an acoustically salient functional category, and qualitatively different components are elicited by structural violations dependent on the form of targets, a result not predicted by 'syntax only' hypotheses of ELAN.

\section{REFERENCES}

Dikker, S., Rabagliati, H., \& Pylkkänen, L. (2009). Sensitivity to syntax in visual cortex. Cognition, 110(3), 293-321. Available from http://dx.doi.org/10.1016/j.cognition.2008.09.008

Friederici, A. D. (2002, 1). Towards a neural basis of auditory sentence processing. Trends in Cognitive Sciences, 6(2), 78-84. Available from http://dx.doi.org/10.1016/S1364-6613(00)01839-8

Friederici, A. D., Pfeifer, E., \& Hahne, A. (1993). Event-related brain potentials during natural speech processing: Effects of semantic, morphological and syntactic violations. Cognitive Brain Research, 1(3), 183-192. Available from http://dx.doi.org/10.1016/0926-6410(93)90026-2

Hestvik, A., Maxfield, N., Schwartz, R., \& Shafer, V. (2007). Brain responses to filled gaps. Brain and Language, $100(3), 301-316$. Available from http://dx.doi.org/10.1016/j.bandl.2006.07.007

Hickok, G., \& Poeppel, D. (2007). The cortical organization of speech processing. Nature Reviews Neuroscience, 8(5), 393-402. Available from http://dx.doi.org/10.1038/nrn2113

Kluender, R., \& Kutas, M. (1993). Bridging the gap: Evidence from ERPs on the processing of unbounded dependencies. Journal of Cognitive Neuroscience, 5(2), 196-214. Available from http://www.mitpressjournals .org/doi/abs/10.1162/jocn.1993.5.2.196

Lau, E., Stroud, C., Plesch, S., \& Phillips, C. (2006). The role of structural prediction in rapid syntactic analysis. Brain and Language, 98(1), 74-88. Available from http://dx.doi.org/10.1016/j.bandl.2006.02.003 